\title{
Weak Bonds in an Aged Cellulose Chromaphore Precursor by Crystallography and Computed Charge Density ${ }^{1}$
}

\author{
Alfred D. French, ${ }^{\mathrm{a}}$ Kurt Mereiter ${ }^{\mathrm{b}}$ and Thomas Rosenau ${ }^{\mathrm{c}}$ \\ a. U.S. Department of Agriculture, Southern Regional Research Center, USDA, New Orleans 70124 \\ b. Department of Chemistry, Vienna University of Technology, Getreidemarkt 9, 1060 Vienna, Austria \\ c. Division of Chemistry of Renewable Resources, Department of Chemistry, University of Natural Resources \\ and Life Sciences (BOKU), Muthgasse 18, 1190 Vienna, Austria
}

A crystal of 1,6-dihydroxy-3,8-bis-hydoxymethyl-5,10-dimethoxy-4,9-dioxa-tricyclo $[5,3,1,1,2,6]$ dodecane-11,12-dione (or $(1,10)$ dimethyl bis (3-keto- $\beta$-D-glucoside) $2,4^{\prime}: 4,2^{\prime}$ dianhydride) grew fortuitously in an NMR tube following studies of 1,4-dimethyl 3-keto $\beta$-Dglucoside in the cellulose solvent N-methylmorpholine N-oxide. The crystal structure revealed that two molecules of the glucoside had fused to create a 10 -membered ring that encompassed two eight-membered rings as well as three six-membered rings.

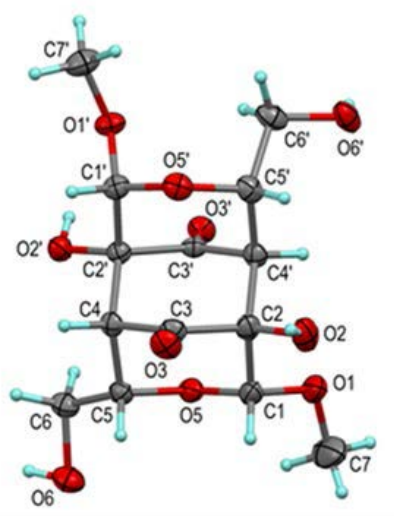

The central six-membered ring is antecedent to 2,5-dihydroxy 1,4benzoquinone, a very powerful chromophore. Besides the unusual spontaneous formation of the compound and the formation of a carbohydrate dianhydride involving oxygens at the 2 and 4 positions on the glucose ring instead of the glycosidic and primary alcohol oxygen atoms, several unusual structural features were observed.

One of the two glucoside rings has the alternate ${ }^{1} \mathrm{C}_{4}$ ring shape while the other has the much more widely found ${ }^{4} \mathrm{C}_{1}$ form. Two questionable intramolecular hydrogen bonds were identified, between the $\mathrm{O} 2-\mathrm{H}$ hydroxyl groups and the $\mathrm{O} 3$ carbonyl groups that did not meet normal length and angle criteria for a hydrogen bond. On the other hand a cyclic hydrogen bond between $\mathrm{O} 6$ groups on adjacent molecules did meet the geometric criteria, at least for the majority positions that were affected by disorder. Because the crystal was not of sufficient quality for a full charge density (electron density topology) analysis, quantum mechanics studies of the electronic structure were carried out at the B3LYP/6-311+G(d,p) level. Neither of the vicinal $\mathrm{O} 2$ hydroxyl and $\mathrm{O} 3$ carbonyl oxygen atoms were connected by a bond path and bond critical point before energy minimization, but the ones on the normal chair were afterwards. The putative cyclic bond showed a sigmoidal bond path between the oxygen atoms but the hydroxyl hydrogen atoms were not involved although there was a broad region of heightened electron density. Also, the calculated O-H stretching frequency was very high, indicating no involvement of the hydrogens. Also detected were bond paths between the carbonyl carbons and the ring oxygen atoms on the opposite sides of the eight-membered, boatshaped rings. The C...O distances were $2.62 \AA$ and $2.64 \AA$. Similar short distances in comparable molecules (one as low as $2.35 \AA$ !) do not usually get comment but have been mentioned by Coppens et al. and Dunitz and colleagues.

\footnotetext{
${ }^{1}$ Published as $2,4^{\prime}: 2^{\prime}, 4$ Dianhydride of 3-keto-glucoside, a precursor to chromophores of aged, yellow cellulose, and its weak interactions. T. Rosenau, A. Potthast, P. Kosma, T. Hosoya, U. Henniges, K. Mereiter, and A. D. French, Cellulose (2017), DOI 10.1007/s10570-017-1198-9
} 